\title{
Identification and Characterization of a Y-Like Primate Retinal Ganglion Cell Type
}

\author{
Dumitru Petrusca, ${ }^{1}$ Matthew I. Grivich, ${ }^{1}$ Alexander Sher, ${ }^{1}$ Greg D. Field, ${ }^{2}$ Jeffrey L. Gauthier, ${ }^{2}$ Martin Greschner, ${ }^{2}$ \\ Jonathon Shlens, ${ }^{2}$ E. J. Chichilnisky, ${ }^{2}$ and Alan M. Litke ${ }^{1}$ \\ ${ }^{1}$ Santa Cruz Institute for Particle Physics, University of California, Santa Cruz, California 95064, and 2The Salk Institute, La Jolla, California 92037
}

\begin{abstract}
The primate retina communicates visual information to the brain via a set of parallel pathways that originate from at least 22 anatomically distinct types of retinal ganglion cells. Knowledge of the physiological properties of these ganglion cell types is of critical importance for understanding the functioning of the primate visual system. Nonetheless, the physiological properties of only a handful of retinal ganglion cell types have been studied in detail. Here we show, using a newly developed multielectrode array system for the large-scale recording of neural activity, the existence of a physiologically distinct population of ganglion cells in the primate retina with distinctive visual response properties. These cells, which we will refer to as upsilon cells, are characterized by large receptive fields, rapid and transient responses to light, and significant nonlinearities in their spatial summation. Based on the measured properties of these cells, we speculate that they correspond to the smooth/large radiate cells recently identified morphologically in the primate retina and may therefore provide visual input to both the lateral geniculate nucleus and the superior colliculus. We further speculate that the upsilon cells may be the primate retina's counterparts of the Y-cells observed in the cat and other mammalian species.
\end{abstract}

Key words: microelectrode array; neural coding; primate; retinal ganglion cell; vision; Y-cell

\section{Introduction}

Although decades of work have been devoted to the study of primate retinal architecture, fewer than one-half of the 22 or more anatomically identified types of retinal ganglion cells (RGCs) (Rodieck and Watanabe, 1993; Dacey et al., 2003, 2005; Dacey, 2004; Yamada et al., 2005) have been characterized physiologically, namely the ON and OFF midget and parasol cells (Dacey, 1999; Chichilnisky and Kalmar, 2002), the small bistratified cells (Dacey and Lee, 1994; Chichilnisky and Baylor, 1999), the recently discovered giant sparse (melanopsin-expressing) cells (Dacey et al., 2003, 2005; Dacey, 2004), and, to some extent, the large bistratified cells and the cells of type "sparse" (Dacey et al., 2003; Dacey, 2004). One of the possible reasons for this discrepancy is that the morphological RGC types awaiting physiological characterization constitute only a small fraction of all the primate ganglion cells (1-4\%) [see Dacey (2004), their table 20.1]. This makes them difficult to encounter and identify with

\footnotetext{
Received June 21, 2007; revised Aug. 29, 2007; accepted Aug. 29, 2007.

This work was supported by National Science Foundation (NSF) Grant PHY-0417175 (A.M.L.), McKnight Foundation (A.M.L., E.J.C.), Alfred P. Sloan Foundation (E.J.C.), National Eye Institute Grant 13150 (E.J.C.), Burroughs Wellcome Fund Career Award at the Scientific Interface (A.S.), Helen Hay Whitney Foundation (G.D.F.), National Institutes of Health National Research Service Award and Chapman Foundation (J.L.G.), German Academic Exchange Service (M.G.), and NSF Integrative Graduate Education and Research Traineeship and La Jolla Interfaces in the Sciences (J.S.). We thank W. Dabrowski, A. A. Grillo, P. Grybos, P. Hottowy, S. Kachiguine, and B. Kutka for their valuable assistance and contributions; S. Barry for machining; H. Fox, K. Osborn, and E. Callaway for access to retinas; and the San Diego Supercomputer Center and the National Science Foundation (cooperative agreements 05253071 and 0438741) for large-scale data storage.

Correspondence should be addressed to Alan M. Litke, Santa Cruz Institute for Particle Physics, Natural Sciences

2, University of California, 1156 High Street, Santa Cruz, CA 95064. E-mail: alan.litke@cern.ch. DOI:10.1523/JNEUROSCI.2836-07.2007

Copyright $\odot 2007$ Society for Neuroscience 0270-6474/07/2711019-09\$15.00/0
}

standard single-electrode recording or small-scale multielectrode array methods.

To search for new physiological cell types in the primate retina, we used a newly developed large-area, high-density multielectrode array system with 512 electrodes, capable of recording the extracellular spiking activity of several hundred RGCs in a single in vitro preparation (Litke et al., 2004). We recorded the RGC activity in isolated pieces of macaque monkey retina, as it responded to a dynamic visual stimulus focused on the photoreceptors.

The main result of these studies is the identification and characterization of a new functional type of primate RGC. These cells have large receptive fields, rapid and transient light responses, and highly nonlinear spatial summation. Cells with similar visual response properties, the Y-cells, were first observed more than forty years ago in the cat retina (Enroth-Cugell and Robson, 1966). The original observation of the cat $Y$-cells was followed by a decades-long search for the counterpart of these cells both in the primate retina and in the primate lateral geniculate nucleus (LGN) (de Monasterio, 1978; Kaplan and Shapley, 1982; Derrington and Lennie, 1984; Blakemore and Vital-Durand, 1986; Benardete et al., 1992; Levitt et al., 2001; White et al., 2002). Until this report, no clear evidence had been found for a distinct type of Y-like retinal ganglion cell in the primate.

\section{Materials and Methods}

Preparations. Eyes were obtained from three deeply and terminally anesthetized macaque monkeys (Macaca mulatta) used by other experimenters, in accordance with institutional guidelines for the care and use of animals. The data reported here came from three retinal preparations (1-3), each from a different macaque monkey. The macaques for prep- 
arations 1 and 3 were experimentally infected with the simian immunodeficiency virus (Roberts et al., 2006); the macaque for preparation 2 was uninfected. No differences were observed in the retinal response properties for the preparations from the infected macaques compared with the uninfected macaque.

Immediately after enucleation, the anterior portion of the eye and vitreous were removed in room light, and the eye cup was placed in bicarbonate buffered Ames' solution (Sigma-Aldrich, St. Louis, MO) and stored in darkness at $32-33^{\circ} \mathrm{C}, \mathrm{pH} 7.4$, for $>20$ min before dissection. Under infrared illumination, pieces of peripheral retina at eccentricities of $\sim 12 \mathrm{~mm}, 3-5 \mathrm{~mm}$ in diameter, isolated from the retinal pigment epithelium, were placed flat, ganglion cell layer down, against a planar array of extracellular microelectrodes. These electrodes were used to record action potentials from spiking retinal cells. Each preparation was perfused with Ames' solution bubbled with $95 \% \mathrm{O}_{2}-5 \% \mathrm{CO}_{2}$, with $\mathrm{pH}$ 7.4. The three preparations (1-3) were maintained, respectively, at $34-$ $35^{\circ} \mathrm{C}, 32-33^{\circ} \mathrm{C}$, and $36-37^{\circ} \mathrm{C}$.

Multielectrode array system. The multielectrode array system has been described by Litke et al. (2004). The electrode array had 512 extracellular microelectrodes arranged in a $32 \times 16$ rectangular pattern, with overall dimensions of $1890 \times 900 \mu \mathrm{m}^{2}$, covering an active area of $1.7 \mathrm{~mm}^{2}$. The electrode-to-electrode spacing was $60 \mu \mathrm{m}$, and each electrode was $5 \mu \mathrm{m}$ in diameter. The analog signal from each electrode was sampled at 20 $\mathrm{kHz}$. This system has been used previously to study the encoding of visual motion and multineuron firing patterns in mosaics of parasol cells in the primate retina (Frechette et al., 2005; Shlens et al., 2006).

Neuron identification. Individual neurons were identified as has been described previously (Litke et al., 2004). Briefly, a spike was identified on an electrode (the "seed" electrode) as a signal amplitude that exceeded a threshold of three times the rms noise. For each spike, a 182-dimensional vector was constructed from the analog waveform measurements on the seed electrode and its six nearest neighbors. Each waveform consisted of the 26 analog samples for the period $0.5 \mathrm{~ms}$ before the spike to $0.8 \mathrm{~ms}$ after the spike. Principal components analysis (PCA) was used to extract the five most significant variables for spike sorting. Multidimensional clustering was performed on these variables, using the expectation maximization algorithm, to find neuron candidates. Duplicate neuron candidates were eliminated by spike train cross-correlation. The absence of a refractory period in the autocorrelation function was used to exclude neuron candidates contaminated by other neurons.

Stimuli. The retina was stimulated with the optically reduced $(2.9 \mathrm{~mm}$ diameter) image of a cathode ray tube display refreshed at $120 \mathrm{~Hz}$, focused on the photoreceptor layer by a microscope objective, and centered on the electrode array. Stimuli were attenuated to low photopic light levels using neutral density filters and presented as modulations around a mean gray background level. The light flux at the gray background level was equivalent to 4400,4300 , and 2200 quanta $\cdot \mu \mathrm{m}^{-2} \cdot \mathrm{s}^{-1}$ produced by monochromatic sources of wavelengths 561, 530, and $430 \mathrm{~nm}$, respectively.

Several visual stimuli were used to classify and characterize each identified neuron. These stimuli included the following: (1) spatiotemporal white noise to measure spatial receptive fields, temporal filtering, and receptive field mosaics; (2) diffuse light steps to measure response kinetics; (3) S-cone-isolating spatiotemporal white noise to evaluate S-cone input; and (4) contrast-reversing gratings (CRGs) to measure the nonlinearity of spatial summation.

The spatiotemporal red, green, and blue (RGB) white noise stimulus consisted of $32 \times 16$ square pixels with each pixel $116 \mu \mathrm{m}$ on a side, refreshed every $8.33 \mathrm{~ms}$. The relative intensity level for each pixel, in each frame, for each of the three color guns (RGB) of the display monitor, was set randomly above or below the 0.5 mean background level at $0.5 \pm 0.48$. The corresponding contrast, $\left(I_{\max }-I_{\min }\right) /\left(I_{\max }+I_{\min }\right)$, was therefore $96 \%$, where $I_{\max }$ and $I_{\min }$ are the maximum and minimum intensities, respectively.

The diffuse light stimulus was a repeating pattern of four full-field frames of white, gray, black, and gray, with relative intensities, respectively, of $0.98,0.5,0.02$, and 0.5 . Each frame had a duration of $2 \mathrm{~s}$, and the contrast was $96 \%$.

The S-cone-isolating S/(L $+\mathrm{M})$ white noise stimulus independently modulated the photon absorption in the S-cones and the summed photon absorptions in the $\mathrm{L}$ and $\mathrm{M}$ cones. This stimulus was obtained by the method of silent substitution (for review, see Estevez and Spekreijse, 1982) and was based on the cone absorption spectra for the macaque monkey (Baylor et al., 1987) and the measured intensity spectrum generated by each of the three color guns. The stimulus contrast was matched at $\sim 40 \%$ for the S-cone-isolating and $(\mathrm{L}+\mathrm{M})$-cone-isolating stimuli. The cross talk between these two stimuli was estimated to be $0 \pm 4 \%$, based on measurements of the light intensity transmitted through filters with filtering characteristics similar to the $\mathrm{S}, \mathrm{M}$, and $\mathrm{L}$ cone absorption spectra. The $S /(L+M)$ white noise stimulus was applied only to the second preparation.

The CRG stimuli were spatial sinusoidal gratings with amplitudes that were sinusoidally modulated in time. The CRG stimuli had a temporal frequency of $4 \mathrm{~Hz}, 10$ spatial frequencies ranging from 0.2 to 28.7 cycles/ $\mathrm{mm}$, and, for each spatial frequency, eight spatial phases ranging from 0 to $315^{\circ}$ in $45^{\circ}$ steps. The contrast for all gratings was $96 \%$.

Spatial and temporal filtering characterization of neurons. The spatial and temporal visual image filtering characteristics of each identified neuron were measured from its spike-triggered average (STA), when the retina was exposed to the RGB white noise stimulus. The STA for each neuron was calculated by averaging, over all spikes generated by the neuron, the sequence of stimulus frames preceding each spike (Sakai et al., 1988; Chichilnisky, 2001).

The receptive field (RF) location, size, and orientation (e.g., see Fig. $3 a$ ) were based on the STA frame most significantly different from the mean (gray) background level. This frame was fit with a two-dimensional generalized Gaussian:

$$
\begin{aligned}
G=A \exp \left[-\frac{1}{2}\left(\frac{\left(x-x_{0}\right) \cos \phi-\left(y-y_{0}\right) \sin \phi}{\sigma_{x}}\right)^{2}\right. \\
\left.-\frac{1}{2}\left(\frac{\left(y-y_{0}\right) \cos \phi+\left(x-x_{0}\right) \sin \phi}{\sigma_{y}}\right)^{2}\right],
\end{aligned}
$$

where $A$ is the amplitude, $x_{0}$ and $y_{0}$ are the locations of the Gaussian center, $\sigma_{x}$ and $\sigma_{y}$ are the semi-axes, and $\phi$ is the orientation angle of the one $\sigma$ contour ellipse. The RF diameter $D$ is defined as the diameter of the circle with the same area as the one $\sigma$ contour ellipse of the Gaussian: $D=$ $2\left(\sigma_{x} \sigma_{y}\right)^{1 / 2}$. The surround was not included in the RF fit because, in most cases, the stimulus used did not significantly activate the surround mechanism in the recorded cells. Furthermore, this representation assumes that the STA is separable in space and time.

The red, green, and blue time filters of each neuron were estimated by the STA time courses corresponding to the three color guns of the stimulus display monitor (e.g., see Fig. $3 f$ ). They were calculated by averaging together the time courses of all STA pixels significantly different from the mean intensity level (a significant pixel was defined as one that differs in absolute value from the mean level by more than three times its rms noise).

Time filter classification parameters. The two time filter classification parameters of each neuron in a collection of neurons (referred to as $\mathrm{PC}_{1}$ and $\mathrm{PC}_{2}$ in Fig. $1 b$ ) were defined as the two most significant principal components obtained by performing PCA on the time filter vectors of all neurons in the collection. The time filter vector for a given neuron was obtained by concatenating its red, green, and blue time filters.

Electrophysiological imaging. The electrophysiological image (EI) is a dynamic image of the average electrical activity of a neuron at the time it generates a spike, projected onto the two-dimensional plane of the electrode array (Litke et al., 2004). The EI reveals the signals generated by the cell body and the dendritic arbor and shows the signal propagation down the axon(s). It is calculated by averaging the analog waveforms recorded on each of the 512 electrodes every time the neuron generates a spike. The EI was used to categorize the identified neurons as either ganglion cells, if they possessed a single axon, or polyaxonal amacrine cells, if they possessed an axonal arbor.

For some cells, no axon or axonal arbor was detected. In all cases, these cells were located close to the border of the electrode array, in a position such that a ganglion cell's axon would be outside of the array's active area. 

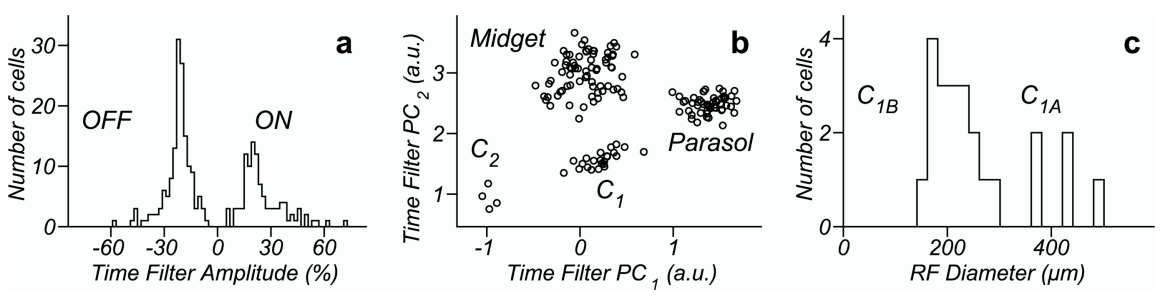

Figure 1. Classification of the 257 cells identified in preparation 1. $\boldsymbol{a}$, Histogram of the amplitude of the primary peak (the peak closest to 0 on the time axis) of the sum of the red, green, and blue time filters. The amplitude is expressed as an intensity change above or below the mean background level (MBL) as a percentage of the MBL. Cells with negative amplitudes are classified as OFF cells, and the cells with positive amplitudes are classified as $0 \mathrm{~N}$ cells. $\boldsymbol{b}$, Classification of $0 \mathrm{FF}$ cells into parasol, midget, $C_{1}$, and $C_{2}$ cell types based on the first two principal components, $\mathrm{PC}_{1}$ and $\mathrm{PC}_{2}$, of their time filters (see Materials and Methods). $c$, Classification of the $C_{1}$ type into two subtypes, $C_{1 A}$ and $C_{1 B}$, based on the RF diameter. a.u., Arbitrary units. the clusters were identified as parasol and midget cells by their RF diameters and their response kinetics (Chichilnisky and Kalmar, 2002). The two remaining clusters, $\mathrm{C}_{1}$ and $\mathrm{C}_{2}$, were of unknown cell type.

The cluster $\mathrm{C}_{2}$ contains a small number of cells. Moreover, this cluster does not represent a unique cell type; the cells it contains differ from each other if additional classification criteria (autocorrelation function, response to contrastreversing gratings, and response to diffuse light steps) are used. Therefore, we will not discuss these cells further.

The neurons in cluster $\mathrm{C}_{1}$ can be divided into two separate clusters, $\mathrm{C}_{1 \mathrm{~A}}$ and Moreover, these cells had the same physiological properties as the cells in the same classification cluster that possessed clearly visible single axons (see the functional classification of neurons in Results). Therefore, we concluded that these cells were ganglion cells.

Response latency. As a measure of the RF center response latency for an RGC, we used the zero crossing time ( $\left.t_{\text {zero }}\right)$ of the STA time filters (Chichilnisky and Kalmar, 2002). In a linear-nonlinear response model (Chichilnisky, 2001), when the cell is stimulated by a small-amplitude light step presented over the RF center, $t_{\text {zero }}$ corresponds to the time from the onset of the light step to the peak of the response.

The calculation of $t_{\text {zero }}$ was based on a fit of the time filter $T$ of each neuron as a sum of two filters:

$$
\begin{aligned}
T(t)=a_{1}\left[\left(t / \tau_{1}\right) \exp \left(1-t / \tau_{1}\right)\right]^{n_{1}}+a_{2}\left[\left(t / \tau_{2}\right) \exp \right. \\
\left.\quad\left(1-t / \tau_{2}\right)\right]^{n_{2}},
\end{aligned}
$$

where $t$ is the time before a spike, $\tau_{1}$ and $\tau_{2}$ are the time constants of the two filters, $a_{1}$ and $a_{2}$ are their amplitudes, and $n_{1}$ and $n_{2}$ are the number of stages of the filters. This function fits all time filters well.

The green time filter was used for the $t_{\text {zero }}$ calculation, because it provides the best measurement as a result of the high signal amplitude. The red or blue time filters can be used instead without any significant difference in the results.

\section{Results}

\section{Functional classification of neurons}

The data shown in this study were recorded from three retinal preparations (1-3) obtained from three different macaque monkeys (see Materials and Methods). Individual neurons were identified in each preparation and were classified into separate functional cell types, based on their responses to a randomly flickering white noise checkerboard stimulus.

Figure $1 a-c$ illustrates the functional classification technique applied to the 257 neurons identified in preparation 1. (Neurons in the other two preparations were classified using similar techniques.) The neurons were first classified as ON-center or OFFcenter, based on the sign of the amplitude of the primary peak of the sum of the red, green, and blue STA time filters (the peak closest to zero on the time axis); the amplitude histogram is shown in Figure $1 a$. The cells with positive amplitudes are classified as ON cells, whereas the cells with negative amplitudes are classified as OFF cells. Only the OFF cells will be subject to additional analysis in the remainder of this paper.

The OFF cells, in turn, were classified into four distinct clusters, shown in Figure $1 b$, according to the detailed shape of their time filters. The shape of each time filter was parameterized by the two most significant principal components, obtained by performing principal components analysis on the combined time filters of all OFF neurons (see Materials and Methods). Two of
$\mathrm{C}_{1 \mathrm{~B}}$, by their RF diameters (Fig. $1 c$ ). The cells in these clusters could be ganglion cells or, possibly, spiking amacrine cells (Stafford and Dacey, 1997; Volgyi et al., 2001). A ganglion cell can be identified by the presence of a single axon. A polyaxonal spiking amacrine cell, on the other hand, can be identified by the presence of an extensive axonal arbor. To image the axonal structure of each neuron, and thereby make possible the separation of ganglion cells from polyaxonal spiking amacrine cells, we used EI analysis (Litke et al., 2004) that provides a detailed spatiotemporal image of the electrical activity of the neuron (see Materials and Methods).

The EIs show that the neurons in the cluster $\mathrm{C}_{1 \mathrm{~A}}$ possess an axonal structure consistent with that expected for ganglion cells. In contrast, the EIs reveal that the neurons associated with cluster $\mathrm{C}_{1 \mathrm{~B}}$ do not have single axons but instead have an axonal arbor structure compatible with that expected for polyaxonal spiking amacrine cells. Example EIs of a parasol cell, a member of the $\mathrm{C}_{1 \mathrm{~A}}$ cluster (later termed an upsilon cell), and a member of the $\mathrm{C}_{1 \mathrm{~B}}$ cluster, are shown in Figure 2. The dynamic electrical activity of these cells can also be viewed with animated versions of their EIs (supplemental movies, available at www.jneurosci.org as supplemental material).

\section{Visual response properties of the upsilon cells}

In what follows, only the members of the $\mathrm{C}_{1 \mathrm{~A}}$ ganglion cell cluster will be considered. We examine the characteristics of their spatial RFs, response kinetics (including response to diffuse light steps and temporal filtering properties), and the linearity of spatial summation. Corresponding analyses are performed on the other two preparations. (An additional study of the strength of the S-cone inputs to the upsilon cells was performed only with the second preparation.)

Based on the uniformity of their properties, as indicated below, both within each preparation as well as from preparation to preparation, we infer that the cells in the $\mathrm{C}_{1 \mathrm{~A}}$ cluster (and the corresponding cells in preparations 2 and 3 ) constitute a single physiological type. Additional evidence for a single cell type, based on the RF mosaics, will also be provided. We will refer to this RGC type as "upsilon." (This name choice will be explained in the discussion section.)

\section{Receptive field diameters and mosaics}

Figure $3 b-d$ shows, for each preparation, the RF mosaics and average RF diameters of the RGCs identified as OFF parasol cells or OFF upsilon cells. The average ratio between the RF diameters of the OFF upsilon and OFF parasol cells is $2.9 \pm 0.4$ (mean \pm $\mathrm{SD})$. The RFs of the parasol cells as well as the upsilon cells form 
almost complete mosaics; the few holes are possibly caused by small inefficiencies in our recording methods. The presence of these upsilon mosaics with nonoverlapping RFs lends additional support to the idea that the upsilon cells constitute a single physiological (and presumably morphological) ganglion cell type (Peichl, 1991).

\section{Response kinetics}

As a first step in characterizing the response kinetics, we recorded the response of each cell to a repeating pattern of diffuse light steps, with intensity increments or decrements in $2 \mathrm{~s}$ intervals (Fig. 3e) (see Materials and Methods). The parasol cells responded with a strong, transient increase in spike rate to light intensity decrements (as expected for an OFF-type cell) and had a weak response to light intensity increments. In addition, the spike rate did not return to its previous value after the transient responses, indicating that the parasol cells possess both transient and sustained responses. (A sustained cell responds to a light step with a change in spike rate for the $2 \mathrm{~s}$ duration of the light step.)

The upsilon cells responded strongly to decrements in light intensity with a transient increase in spike rate but did not respond at all to light intensity increments.

In contrast to parasol cells, the spike rate returned to its previous resting discharge rate (mean $\pm \mathrm{SD}=0.1 \pm 0.2 \mathrm{spikes} / \mathrm{s}$ for the data shown in Fig. 3e, right) after the transient responses, indicating that the upsilon cells are highly transient.

The temporal filtering properties of the parasol and upsilon cells (in preparation 1) are indicated by the STA time filters shown in Figure $3 f$. As a measure of each neuron's response latency, we have used $t_{\text {zero }}$ of the STA time filter (Chichilnisky and Kalmar, 2002) (see Materials and Methods). The average $t_{\text {zero }}$ values (mean $\pm \mathrm{SD}$ ) for the parasol cells in preparations $1-3$ are, respectively, $53.8 \pm 2.0,75.4 \pm 3.8$, and $50.2 \pm 3.8 \mathrm{~ms}$, with $n=$ 52,86 , and 114 . The corresponding values for the upsilon cells are $67.3 \pm 3.6,70.1 \pm 4.9$, and $56.1 \pm 2.6 \mathrm{~ms}$, with $n=5,10$, and 8 . For completeness, we include also the $t_{\text {zero }}$ values for the OFFmidget cells in the three preparations; these are $80.5 \pm 7.5$, 106.9 \pm 6.7 , and $76.2 \pm 8.8 \mathrm{~ms}$, with $n=67,15$, and 28 . The ratio of the average $t_{\text {zero }}$ for the upsilon cells to the average $t_{\text {zero }}$ for the parasol cells, averaged over the three preparations, is $1.10 \pm 0.16$ (mean $\pm \mathrm{SD}$ ). The corresponding upsilon-to-midget ratio is $0.74 \pm 0.09$. Therefore, the upsilon cells have response latencies similar to those of the simultaneously recorded parasol cells but shorter than those of the simultaneously recorded midget cells.

\section{Color response properties: S-cone inputs}

Because of recent observations indicating that some large-field ganglion cells possess selective connectivity to S-cones (Dacey and Packer, 2003; Dacey et al., 2003, 2005; Dacey, 2004), we investigated the $\mathrm{S}$-cone input to the upsilon cells by using the cone-isolating $\mathrm{S} /(\mathrm{L}+\mathrm{M})$ stimulus (see Materials and Methods). The response of the upsilon cells to this stimulus was dominated b

c
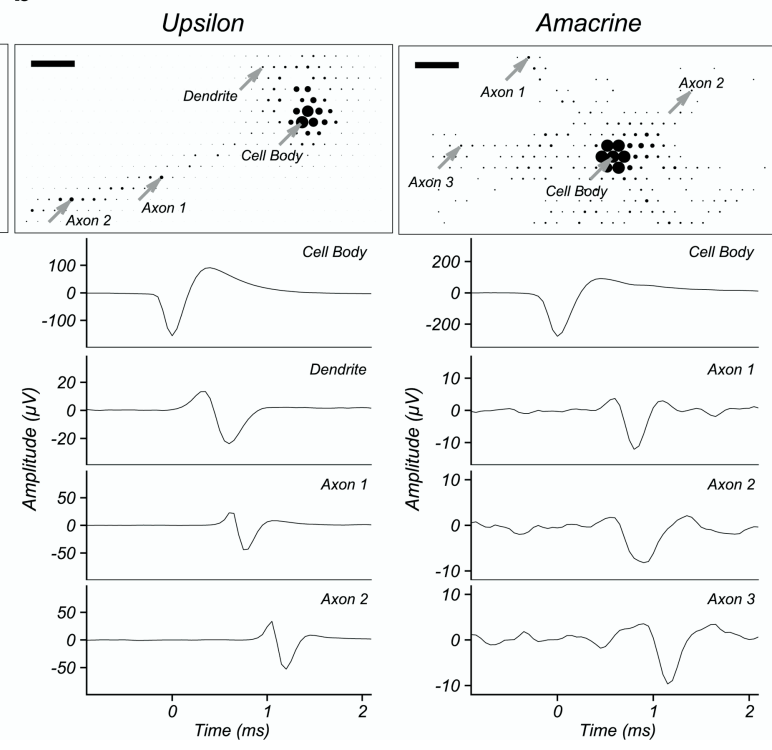
signals. The axonal signals are characterized by their triphasic shapes and by the displacement in time as a result of signal member of the $C$ cluster; see Results). $c$ El and signal shapes for a spiking amacrine cell (a member of the ately the same for the parasol and upsilon cells. Scale bars, $200 \mu \mathrm{m}$. For clarity, the El circle diameters in c (top) were truncated by the $(\mathrm{L}+\mathrm{M})$-cone OFF component, as shown in Figure 4. These data indicate that the S-cone input to the upsilon cells is a very small fraction of their total input. This fraction is measured to be $4.4 \pm 4.1 \%$, based on the peak of the S-cone time filter response relative to the peak of the $\mathrm{S}$-cone plus $(\mathrm{L}+\mathrm{M})$-cone response, and including an estimated cross talk of $0 \pm 4 \%$ between the $S$ and $(\mathrm{L}+\mathrm{M})$ cone-isolating stimuli. For comparison, the corresponding S-cone fraction measured for S-ON/(L + M)OFF cells [most likely small bistratified blue/yellow color opponent cells (Dacey and Lee, 1994)] in the same preparation is $48.4 \pm 16.6 \%$, indicating that the S-cones were functioning normally (data not shown). (This insignificant S-cone input to upsilon cells, measured in the presence of simultaneously recorded $\mathrm{S}-\mathrm{ON} /(\mathrm{L}+\mathrm{M})$-OFF cells, was also observed in several preparations that contained only a few upsilon cells and therefore were not included in this paper.) The combination of a strong ( $\mathrm{L}+$ $\mathrm{M}$ )-cone OFF response and weak-to-negligible S-cone OFF response differentiates the upsilon cells from the large bistratified, sparse, and giant sparse (melanopsin-expressing) large-field cells, all with significant S-cone/(L $+\mathrm{M})$-cone opponent responses (Dacey and Packer, 2003; Dacey et al., 2003, 2005; Dacey, 2004).

\section{Linearity of spatial summation}

To gain insight into how the visual input signals get summed spatially by the upsilon cells, we have investigated their spatial summation properties and compared these properties with those of the midget and parasol cells. An RGC that sums the visual input linearly over its RF is known as an X-like cell, whereas an RGC that exhibits nonlinear spatial summation is known as a Y-like cell (Enroth-Cugell and Robson, 1966). It is thought that the receptive fields of Y-like cells are constructed by pooling ex- 


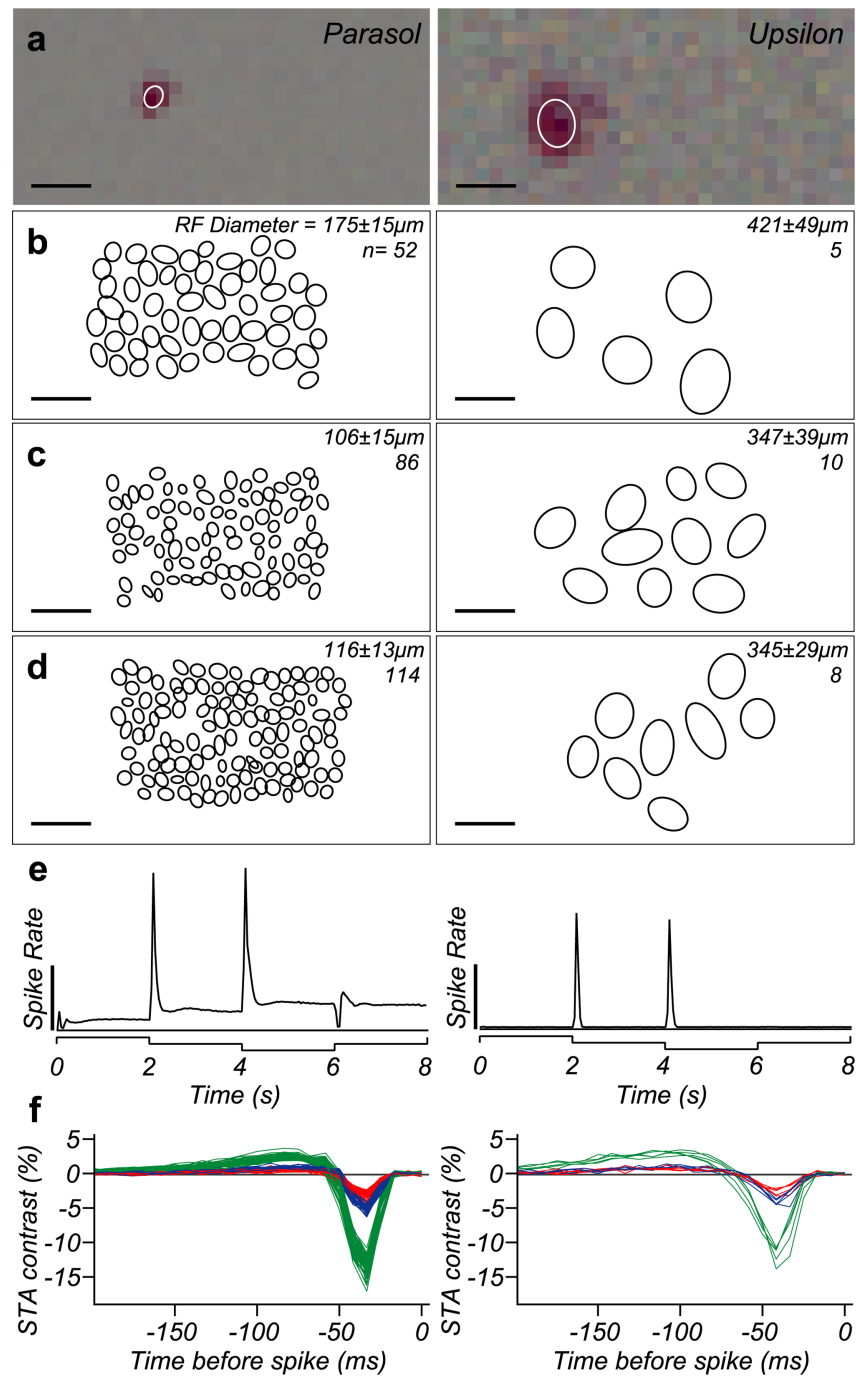

Figure 3. Parasol and upsilon ganglion cell properties. $\boldsymbol{a}$, Typical RF STA for an OFF parasol cell (left) and an OFF upsilon cell (right) in preparation 1. The 1 SD contour ellipses of the two-dimensional Gaussian fits to the spatial STAs are shown. $\boldsymbol{b}$ - $\boldsymbol{d}$, RF mosaics of OFF parasol (left) and OFF upsilon (right) cells in preparations 1-3, respectively. The average RF diameter (mean \pm SD) and the number of cells $(n)$ for the cells in each mosaic are indicated in the top right corner of each panel (see Materials and Methods). Scale bars, $500 \mu \mathrm{m} . e$, Average parasol and upsilon cell responses to diffuse light steps in preparation 1. The horizontal lines define the zero spike rates. The traces below the plots show the time course of the 8-s-long repeating light stimulus. Calibration, 100 spikes/s. Both cell types responded strongly to light intensity decrements and weakly or not at all to light intensity increments. $f$, Red, green, and blue time filters for OFF parasol (left) and OFF upsilon (right) cells in preparation 1, corresponding to the three color guns of the display monitor. The time filters are sampled each $8.33 \mathrm{~ms}$, which corresponds to the refresh interval of the display monitor. The STA contrast is expressed as an intensity change above or below the mean background level (MBL) as a percentage of the MBL.

citation from an array of small nonlinear spatial subunits (Hochstein and Shapley, 1976b; Shapley and Victor, 1979; Victor and Shapley, 1979; Demb et al., 1999, 2001a), and it has been proposed that these subunits are bipolar cells (Victor and Shapley, 1979; Demb et al., 1999, 2001a).

We have studied this nonlinearity in the classical way by recording the responses of neurons to CRGs (Hochstein and Shapley, 1976a,b; Derrington and Lennie, 1984). The CRG is a spatial sinusoidal grating with a contrast that is modulated sinusoidally in time (see Materials and Methods). Midget, parasol, and upsilon cell average responses to one period of low and high spatial frequency CRGs at four spatial phases, in preparation 1, are shown in Figure $5 a-c$, respectively.

The responses to the CRGs of low spatial frequency (0.2 cycles $/ \mathrm{mm}$ ) are modulated in time mainly at the temporal frequency of the stimulus (implying a significant first Fourier harmonic, $\left.F_{1}\right)$, because at low spatial frequencies, all the subunits are stimulated in phase. Additionally, the $F_{1}$ response amplitude varies sinusoidally as a function of the spatial phase of the CRG, with null responses at two spatial phases separated by $180^{\circ}$ (shown as 90 and $270^{\circ}$ in Fig. $5 a-c$ ).

At high spatial frequency ( 4.8 cycles $/ \mathrm{mm})$, the midget cells still respond mainly at the temporal frequency of the stimulus, indicating that spatial summation in their RFs remains dominantly linear (that is, they exhibit X-like behavior). The upsilon cells, however, display a response modulated mainly at twice the temporal frequency of the stimulus (two peaks per stimulus period, corresponding to a significant second Fourier harmonic, $F_{2}$; this is the classical signature for Y-like cells). This frequency doubling response may arise because approximately one-half of the nonlinear subunits are stimulated in counterphase relative to the other half of the subunits, assuming the nonlinearity is half-wave rectification [as supported for Y-cells by experimental data (Demb et al., 2001a)]. This nonlinear spatial summation leads to an $F_{2}$ response only weakly dependent on spatial phase, as can be seen in Figure $5 c$ (right column).

The parasol cells also show an $F_{2}$ response at the high spatial frequency $(4.8$ cycles $/ \mathrm{mm}$ ), as is apparent in Figure $5 b$ (right column). However, the $F_{2}$ response coexists with an $F_{1}$ response of similar amplitude, which explains the stronger dependence on spatial phase.

Figures $5 a-c$ illustrate qualitatively the different nonlinear response properties of single midget, parasol, and upsilon cells at two spatial frequencies. To more completely characterize and quantify the nonlinear spatial summation response properties of these cell types, we measured the $F_{1}$ and $F_{2}$ Fourier harmonics, as a function of spatial frequency, for all the midget, parasol and upsilon cells in the three preparations. The harmonics of the response of each cell, under the different CRG stimuli (see Materials and Methods), were calculated by temporal Fourier transforms (Hochstein and Shapley, 1976a). The spatial frequency dependence of the $F_{1}$ and $F_{2}$ responses were obtained after taking account of the phase dependence. This was done by taking the maximum value of $F_{1}$ and the mean value of $F_{2}$ over all spatial phases (Hochstein and Shapley, 1976a). Figure $5 d-f$ shows the spatial frequency tuning curves of the average $F_{1}$ and $F_{2}$ harmonics for the three cell types in each of the three preparations.

The midget cell responses are dominated by the $F_{1}$ harmonic at all measured spatial frequencies (up to 28.7 cycles $/ \mathrm{mm}$ ) (Fig. $5 d$ ), indicating that they are X-like cells in terms of spatial summation. In contrast, both parasol and upsilon cells have large $F_{2}$ harmonics at high spatial frequencies (i.e., they show frequency doubling responses) (Fig. 5e,f) and therefore possess significant nonlinearities in their spatial summation.

We have quantified the level of nonlinearity of spatial summation with the maximum value of the ratio between the second and the first Fourier harmonic $\left(F_{2} / F_{1}\right)$ over all spatial frequencies recorded. This value is called the nonlinearity index (Hochstein and Shapley, 1976a).

Histograms of the nonlinearity indices for midget, parasol, and upsilon cells are shown in Figure 6. These histograms show significant overlap between the nonlinearity indices of midget and parasol cells and also between those of parasol and upsilon cells. However, on average, the parasol cells have $\sim 3.8$ times 
larger nonlinearity indices than the midget cells, and the upsilon cells have $\sim 2.6$ times larger nonlinearity indices than the parasol cells. In summary, we find that the upsilon cells have Y-like properties, with the nonlinear spatial summation response $\left(F_{2}\right)$ dominating the linear response $\left(F_{1}\right)$ over a broad range of spatial frequencies.

\section{Comparison of the nonlinearity index results with previous studies}

In this subsection, we will compare the nonlinearity index distributions, as shown in Figure 6, with the previously measured results in macaque retina (White et al., 2002) and in macaque LGN (Derrington and Lennie, 1984; Levitt et al., 2001). The parasol and upsilon nonlinearity distributions shown in Figure 6 have long tails that extend to 12-13. These are different from the distributions shown by Derrington and Lennie (1984), Levitt et al. (2001), and White et al. (2002), which extend only up to values of $2-4$. We think this discrepancy is attributable to differences in the methods used to calculate the nonlinearity index.

We followed the method suggested by Hochstein and Shapley (1976a) and defined the nonlinearity index as the maximum value of the $F_{2} / F_{1}$ ratio over a wide range of spatial frequencies. In the experiments of Derrington and Lennie (1984) and White et al. (2002), the nonlinearity index was calculated at 1.5-2 times the spatial frequency of maximum response. However, the experimental data for parasols shown in Figure $5 e$ indicate that the peak response is $\sim 2$ cycles $/ \mathrm{mm}$ and that measuring the nonlinearity index at twice this frequency $(4$ cycles $/ \mathrm{mm})$ falls short of the most nonlinear frequency domain $(\sim 7$ cycles $/ \mathrm{mm})$. Likewise, for the upsilon data shown in Figure $5 f$, the peak response is in the range $0.3-1$ cycles $/ \mathrm{mm}$, and measuring the nonlinearity index at twice these values $(0.6-2$ cycles $/ \mathrm{mm})$ again falls short of the most nonlinear region (3-7 cycles $/ \mathrm{mm})$. By restricting the nonlinearity index measurement to spatial frequencies not sufficiently high, the nonlinearity indices were possibly underestimated.

In the experiment of Levitt et al. (2001), spatial frequencies that had small $F_{1}$ responses were ignored, even when there was a significant $F_{2}$ response. Again, this has the potential to underestimate the nonlinearity index. We suggest that these factors may explain the differences between the distributions in this study and the results of the previous studies.

\section{Discussion}

The main result presented in this paper is the identification and characterization of a new physiological type of ganglion cell in the primate retina. We refer to this new type as OFF upsilon. These cells are characterized by large receptive fields (with approximately three times the RF diameter of parasol cells at the same eccentricity), highly transient and rapid OFF responses to diffuse light steps (with insignificant $\mathrm{ON}-\mathrm{OFF}$ responses), the absence of significant S-cone selective input, and highly nonlinear spatial summation. These characteristics can be used to identify these cells and separate them from all other known macaque functional RGCs. With as yet no confirmed morphological counterpart, we have chosen the name upsilon for this cell type to emphasize its Y-like character (the capital Greek letter upsilon is written as a roman "Y"). a

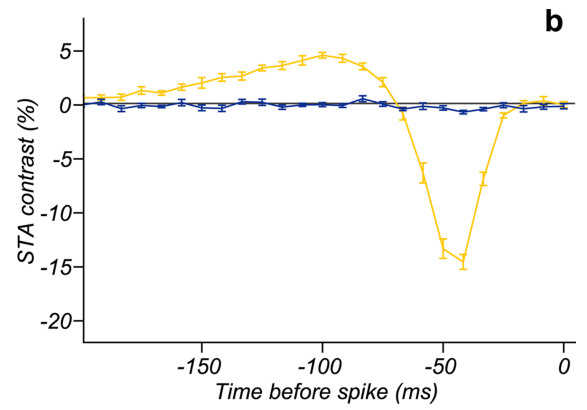

b

Figure 4. S-cone input to the upsilon ganglion cells, based on the cone-isolating $S /(L+M)$ stimulus. The $S$-cone photon absorption and the sum of the photon absorptions in the $L$ and $M$ cones were independently modulated (see Materials and hods). $\boldsymbol{a}$, S-cone-isolating (blue) and (L + M)-cone-isolating (yellow) STA time filters for OFF upsilon cells in preparation 2. $\boldsymbol{b}$, refresh interval of the display monitor. The STA contrast is expressed as an intensity change above or below the mean background

\section{The role of large-scale multielectrode array recording}

The large-scale multielectrode array recording system (Litke et al., 2004) had a combination of features of particular importance for these investigations. These features included a high density of electrodes (300 electrodes $/ \mathrm{mm}^{2}$ ), fine time resolution (50 $\mu \mathrm{s}$ sampling interval) and large active area $\left(1.7 \mathrm{~mm}^{2}\right)$. The combination of these features contributed to the reported results in the following critical ways: (1) the efficient identification of neurons (spike sorting) based on the correlated analog waveforms recorded on multiple nearby electrodes; (2) the ability to separate ganglion cells from spiking amacrine cells by a dynamic highdensity EI of the electrical activity of each identified neuron. The EI also provided a valuable check on the quality of neuron identification; (3) the detection of a sufficiently large sample of neurons of each type to allow statistically significant clustering for neuron classification; and (4) the ability to detect enough cells of a given type, over a sufficiently large area, to observe and characterize a mosaic, even for cells with large receptive fields.

\section{Possible morphological correlate of the upsilon cells}

Based on the measured upsilon cell properties, we can speculate as to which morphological RGC type (Rodieck and Watanabe, 1993; Dacey et al., 2003, 2005; Dacey, 2004; Yamada et al., 2005) they may correspond to. Because the upsilon cells are OFF-type cells with no evidence for ON-OFF responses (i.e., no significant responses to both light intensity increments and decrements) (Fig. 3e), we expect that they would correspond to a cell whose dendrites narrowly stratify in the outer part of the inner plexiform layer (Nelson et al., 1978; Peichl and Wassle, 1981). Also, because the upsilon cells do not show any significant S-cone/(L + $\mathrm{M}$ )-cone opponent response properties, we do not expect them to correspond to the sparse or giant sparse (melanopsinexpressing) cells (Dacey and Packer, 2003; Dacey, 2004; Dacey et al., 2005). Furthermore, because the ratio of the RF diameter of the OFF upsilon cells to the RF diameter of OFF parasol cells is $\sim 2.9$, the ratio of the dendritic arbor diameter of the upsilon cells to the dendritic arbor diameter of the parasol cells is expected to also be $\sim 2.9$ [assuming the same ratio of the RF to the dendritic arbor diameters for both cell types (Peichl and Wassle, 1983)]. Given these constraints, the most plausible morphological candidate(s) corresponding to the upsilon cells are the smooth (Dacey, 2004) and large radiate (Yamada et al., 2005) ganglion cells recently identified in the primate retina.

Both the smooth and large radiate cells exist as inner and outer 


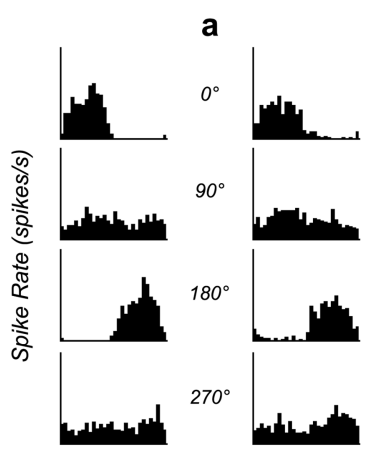

Midget
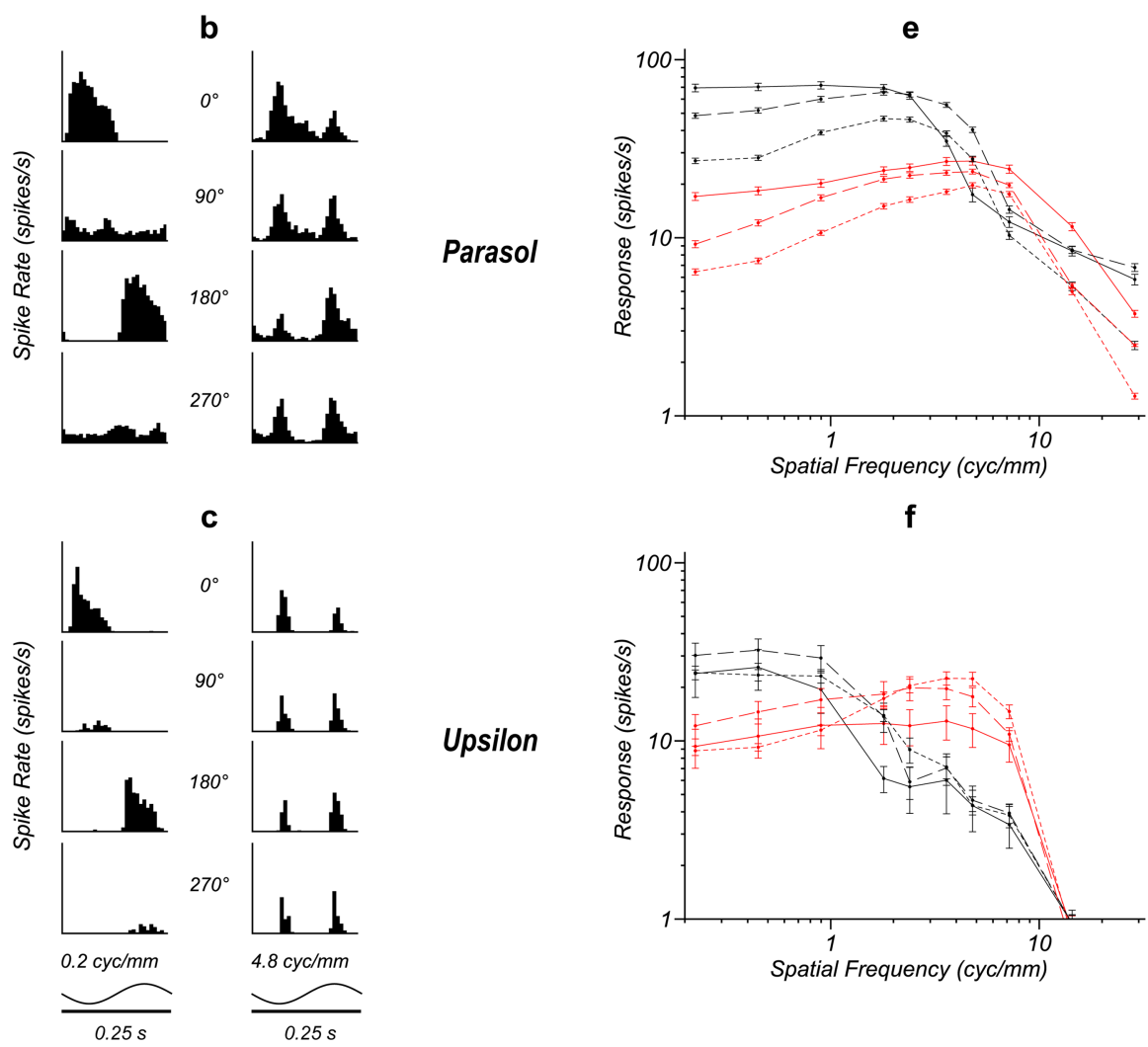

Upsilon

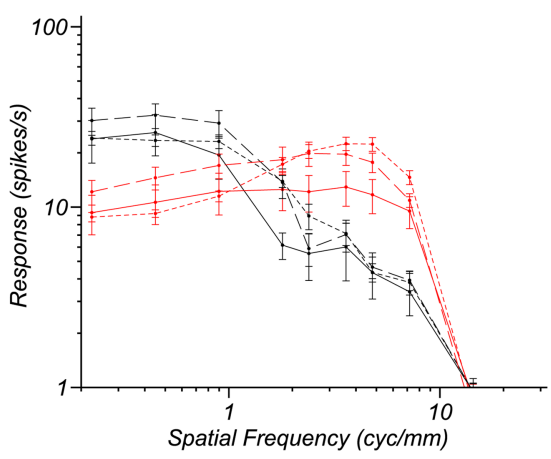

Figure 5. Responses of midget, parasol, and upsilon ganglion cells to CRGs of different spatial frequencies. $\boldsymbol{a}-\boldsymbol{c}$, Typical responses to CRG stimulation of a midget $(\boldsymbol{a})$, parasol $(\boldsymbol{b})$, and upsilon (c) cell at two spatial frequencies $(0.2$ and $4.8 \mathrm{cycles} / \mathrm{mm})$ and four spatial phases $\left(0,90,180\right.$, and $\left.270^{\circ}\right)$ in preparation 1 . The temporal (horizontal) scale and stimulus period of $250 \mathrm{~ms}$ are indicated by the solid bar and single sinusoidal temporal cycle below each column. The vertical scales are 100, 250, and 150 spikes/s in $\boldsymbol{a}-\boldsymbol{c}$, respectively. $\boldsymbol{d}-\boldsymbol{f}$, Spatial frequency tuning curves for the first Fourier harmonic $\left(F_{1} ;\right.$ black $)$ and the second Fourier harmonic $\left(F_{2}\right.$; red) response amplitudes of midget $(\boldsymbol{d})$, parasol $(\boldsymbol{e})$, and upsilon $(\boldsymbol{f})$ cells in preparation 1 (solid lines), 2 (dotted lines), and 3 (dashed lines). $F_{1}$ is calculated as the maximum and $F_{2}$ as the mean harmonic of the responses of the cells over all spatial phases. The error bars represent the SEM.

stratifying subtypes with consistent stratification levels and with very similar dendritic tree structures. Furthermore, both cell types are morphologically similar (Dacey, 2004; Yamada et al., 2005 ) to the intensively studied cat $\alpha$-cells (Boycott and Wassle, 1974; Wassle et al., 1981). Therefore, in what follows, we will consider them as a single cell type that has been given different names by different investigators. We will refer to them as smooth/ large radiate cells.

The upsilon cells may be the primate counterpart of the Ycells of the cat retina

Given the morphological similarity of the primate smooth/large radiate cells to the cat $\alpha$-cells (Dacey, 2004; Yamada et al., 2005), and given that the physiological counterpart of the cat $\alpha$-cell is the Y-cell (Cleland et al., 1975; Peichl and Wassle, 1981), it may be expected that the smooth/large radiate cells will possess physiological properties similar to the Y-cells of the cat, namely rapid and transient light responses, large receptive fields, and nonlinear spatial summation. The fact that these visual response properties also characterize the upsilon cells reported here brings full circle the following physiological/morphological correspondences: primate upsilon cells $\rightarrow$ primate smooth/large radiate cells $\rightarrow$ cat $\alpha$-cells $\rightarrow$ cat Y-cells $\rightarrow$ primate upsilon cells. This suggests that the upsilon (smooth/large radiate) cells may be the primate retina's physiological (morphological) counterparts of the $\mathrm{Y}(\alpha)$ ganglion cells of the cat retina and the retinas of other mammalian species (Peichl, 1991).

If the upsilon (smooth/large radiate) correspondence is correct, then the upsilon cells would project to both the LGN and the superior colliculus (SC), as observed for the smooth cells by retrograde labeling experiments (Dacey, 2004). This property of projection to both the LGN and the SC is also a property of the $\mathrm{Y}(\alpha)$-cells of the cat (Wassle and Illing, 1980), further strengthening the upsilon (smooth/large radiate)to-Y $(\alpha)$ correspondence.

\section{$\mathrm{X} / \mathrm{Y}$-cell classification}

As mentioned in the introduction, there has been a decades-long search for primate Y-cells in both the retina and the LGN. The data of Kaplan and Shapley (1982) in macaque LGN indicated the existence of two types of cells: magnocellular X $\left(\mathrm{M}_{\mathrm{X}}\right)$-cells, showing linearity in spatial summation, and nonlinear magnocellular $\mathrm{Y}\left(\mathrm{M}_{\mathrm{Y}}\right)$-cells. These results supported the hypothesis that the $\mathrm{M}_{\mathrm{Y}}$-cells form a functional group with properties distinct from the $\mathrm{M}_{\mathrm{X}}$-cells (hypothesis 1). However, the measurements of Kaplan and Shapley (1982) did not use a quantitative description of nonlinearity, as introduced, for example, by Hochstein and Shapley (1976a). This makes it difficult to determine whether the $\mathrm{M}_{\mathrm{X}^{-}}$and $\mathrm{M}_{\mathrm{Y}}$-cells are indeed distinct cell types or, instead, represent two ends of a nonlinearity distribution for a single cell type (hypothesis 2).

Later studies in macaque LGN (Derrington and Lennie, 1984; Levitt et al., 2001) and in macaque retina (White et al., 2002) used the quantitative nonlinearity index of Hochstein and Shapley (1976a) to search for distinct X- and Y-like cell types. However, the measured nonlinearity distributions were all unimodal, in support of hypothesis 2 but inconsistent with hypothesis 1 .

The data in this study seem to reconcile the two hypotheses. The measurement of the nonlinearity distribution of macaque RGCs as shown in Figure $6 d$, without separation into the parasol and upsilon cell types, shows a unimodal distribution consistent 
a

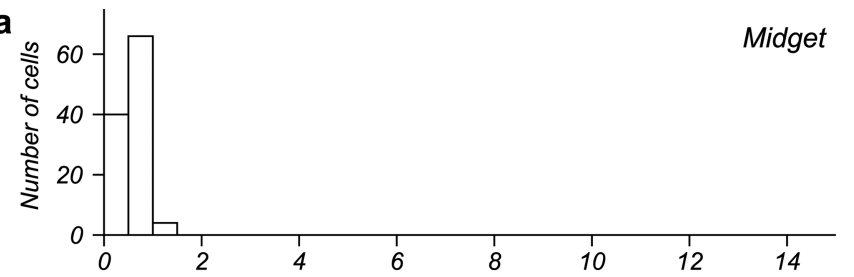

b
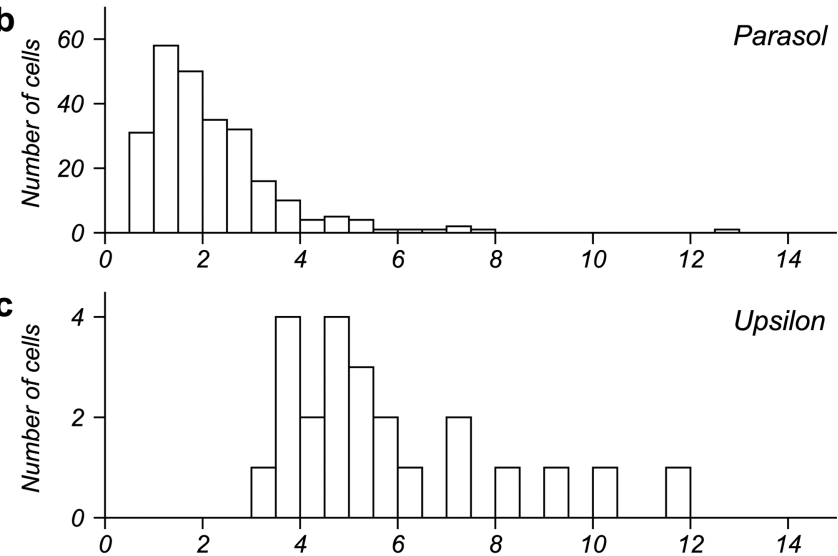

d

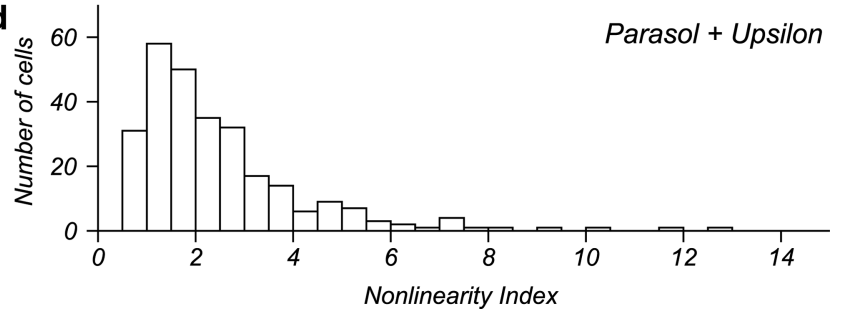

Figure 6. Spatial summation nonlinearities of midget, parasol, and upsilon cells. $\boldsymbol{a}-\boldsymbol{c}$, Nonlinearity index histograms for midget $(\boldsymbol{a})$, parasol $(\boldsymbol{b})$, and upsilon (c) cells in all three preparations; $\boldsymbol{d}$ combines the parasol and upsilon histograms into a single histogram. The nonlinearity index is defined as the maximum value of the ratio between the second and the first harmonics $\left(F_{2} / F_{1}\right)$ over all spatial frequencies recorded. The average values of the nonlinearity indices are $0.6 \pm 0.2(n=110)$ for midget cells, $2.2 \pm 1.4(n=252)$ for parasol cells, and $5.8 \pm 2.2(n=$ 23) for upsilon cells (mean \pm SD). Note that the cell with the highest nonlinearity index is a parasol cell with an index of $12.8(\boldsymbol{b})$. This cell is one outlier in a population of 252 parasol cells. It has a robust $F_{2}$ amplitude of 39.7 spikes $/$ s, but a low $F_{1}$ amplitude of 3.1 spikes $/ \mathrm{s}$. The parasol and upsilon nonlinearity index distributions both appear to have significant high-end tails. If the upsilon sample size was increased by an order of magnitude to $\sim 250$ cells (the same sample size as the parasols), we would expect the nonlinearity index tail to exceed 13.

with hypothesis 2 . However, if the cells are classified using additional information, such as RF size and time filter characteristics, then one finds two distinct distributions of nonlinearity indices, as shown in Figure $6, b$ and $c$. The distribution of Figure $6 c$, in particular, indicates a distinct type of highly nonlinear Y-like RGCs (the upsilons), consistent with hypothesis 1. [Interestingly,

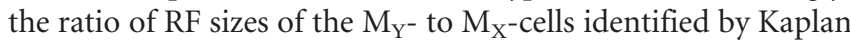
and Shapley (1982), based on the response to a drifting sine wave grating as a function of spatial frequency, is 2.6 , consistent with the upsilon-to-parasol RF diameter ratio of $2.9 \pm 0.4$ reported in this study. Of course, the caveat is that we have no direct evidence that the upsilon cells project to the LGN.]

\section{Future investigations}

These results and speculations raise two critical issues for future physiological and anatomical investigations. First, the correspondence of the upsilon cells with the smooth/large radiate cells needs to be confirmed through combined physiological and morphological experiments. Second, if the OFF upsilon cells are in- deed the physiological counterparts of the outer stratifying smooth/large radiate cells, one should also find the physiological correlates of the inner stratifying smooth/large radiate cells (i.e., the ON upsilon cells). Our preliminary data on the ON upsilon cells suggest that these cells do exist and that they have properties similar to the OFF upsilon cells (i.e., large RFs, rapid and transient light responses, and nonlinear spatial summation). However, because of very limited statistics, more experiments are required to confirm their existence and to study their properties.

\section{Implications}

These findings have the following implications: (1) the rapid, transient, and highly nonlinear spatial summation (Y-like) response properties of the upsilon cells are well suited for the detection of moving objects or moving textured patterns. This includes fine-grained patterns with constant luminance but drifting contrast modulation [second-order motion (Demb et al., 2001b)]. Assuming the upsilon cells project to both the LGN and the SC, they may simultaneously contribute to the primary visual pathway for motion perception and provide the SC with the information needed for triggering attention to movement; (2) the upsilon cells may be the primate counterparts of the $\mathrm{Y}(\alpha)$-cells of the cat (and many other mammalian species) and may originate similar visual processing pathways; and (3) these cells may contribute to nonlinear spatial summation properties observed for some cells recorded in the magnocellular layers of the primate LGN (Kaplan and Shapley, 1982).

\section{References}

Baylor DA, Nunn BJ, Schnapf JL (1987) Spectral sensitivity of cones of the monkey Macaca fascicularis. J Physiol (Lond) 390:145-160.

Benardete EA, Kaplan E, Knight BW (1992) Contrast gain control in the primate retina: $\mathrm{P}$ cells are not X-like, some $\mathrm{M}$ cells are. Vis Neurosci 8:483-486.

Blakemore C, Vital-Durand F (1986) Organization and post-natal development of the monkey's lateral geniculate nucleus. J Physiol (Lond) 380:453-491.

Boycott BB, Wassle H (1974) The morphological types of ganglion cells of the domestic cat's retina. J Physiol (Lond) 240:397-419.

Chichilnisky EJ (2001) A simple white noise analysis of neuronal light responses. Network 12:199-213.

Chichilnisky EJ, Baylor DA (1999) Receptive-field microstructure of blueyellow ganglion cells in primate retina. Nat Neurosci 2:889-893.

Chichilnisky EJ, Kalmar RS (2002) Functional asymmetries in ON and OFF ganglion cells of primate retina. J Neurosci 22:2737-2747.

Cleland BG, Levick WR, Wassle H (1975) Physiological identification of a morphological class of cat retinal ganglion cells. J Physiol (Lond) 248:151-171.

Dacey DM (1999) Primate retina: cell types, circuits and color opponency. Prog Retin Eye Res 18:737-763.

Dacey DM (2004) Origins of perception: retinal ganglion cell diversity and the creation of parallel visual pathways. In: The cognitive neurosciences III (Gazzaniga MS, ed), pp 281-301: Cambridge, MA: MIT.

Dacey DM, Lee BB (1994) The "blue-on" opponent pathway in primate retina originates from a distinct bistratified ganglion cell type. Nature 367:731-735.

Dacey DM, Packer OS (2003) Colour coding in the primate retina: diverse cell types and cone-specific circuitry. Curr Opin Neurobiol 13:421-427.

Dacey DM, Peterson BB, Robinson FR, Gamlin PD (2003) Fireworks in the primate retina: in vitro photodynamics reveals diverse LGN-projecting ganglion cell types. Neuron 37:15-27.

Dacey DM, Liao HW, Peterson BB, Robinson FR, Smith VC, Pokorny J, Yau KW, Gamlin PD (2005) Melanopsin-expressing ganglion cells in primate retina signal colour and irradiance and project to the LGN. Nature 433:749-754.

de Monasterio FM (1978) Properties of concentrically organized X and Y ganglion cells of macaque retina. J Neurophysiol 41:1394-1417.

Demb JB, Haarsma L, Freed MA, Sterling P (1999) Functional circuitry of 
the retinal ganglion cell's nonlinear receptive field. J Neurosci 19:9756-9767.

Demb JB, Zaghloul K, Haarsma L, Sterling P (2001a) Bipolar cells contribute to nonlinear spatial summation in the brisk-transient $(\mathrm{Y})$ ganglion cell in mammalian retina. J Neurosci 21:7447-7454.

Demb JB, Zaghloul K, Sterling P (2001b) Cellular basis for the response to second-order motion cues in Y retinal ganglion cells. Neuron 32:711-721.

Derrington AM, Lennie P (1984) Spatial and temporal contrast sensitivities of neurones in lateral geniculate nucleus of macaque. J Physiol (Lond) $357: 219-240$

Enroth-Cugell C, Robson JG (1966) The contrast sensitivity of retinal ganglion cells of the cat. J Physiol (Lond) 187:517-552.

Estevez O, Spekreijse H (1982) The "silent substitution" method in visual research. Vision Res 22:681-691.

Frechette ES, Sher A, Grivich MI, Petrusca D, Litke AM, Chichilnisky EJ (2005) Fidelity of the ensemble code for visual motion in primate retina. J Neurophysiol 94:119-135.

Hochstein S, Shapley RM (1976a) Quantitative analysis of retinal ganglion cell classifications. J Physiol (Lond) 262:237-264.

Hochstein S, Shapley RM (1976b) Linear and nonlinear spatial subunits in Y cat retinal ganglion cells. J Physiol (Lond) 262:265-284.

Kaplan E, Shapley RM (1982) X and Y cells in the lateral geniculate nucleus of macaque monkeys. J Physiol (Lond) 330:125-143.

Levitt JB, Schumer RA, Sherman SM, Spear PD, Movshon JA (2001) Visual response properties of neurons in the LGN of normally reared and visually deprived macaque monkeys. J Neurophysiol 85:2111-2129.

Litke AM, Bezayiff N, Chichilnisky EJ, Cunningham W, Dabrowski W, Grillo AA, Grivich M, Grybos P, Hottowy P, Kachiguine S, Kalmar RS, Mathieson K, Petrusca D, Rahman M, Sher A (2004) What does the eye tell the brain?: Development of a system for the large-scale recording of retinal output activity. IEEE Trans Nucl Sci 51:1434-1440.

Nelson R, Famiglietti Jr EV, Kolb H (1978) Intracellular staining reveals different levels of stratification for on- and off-center ganglion cells in cat retina. J Neurophysiol 41:472-483.

Peichl L (1991) Alpha ganglion cells in mammalian retinae: common properties, species differences, and some comments on other ganglion cells. Vis Neurosci 7:155-169.

Peichl L, Wassle H (1981) Morphological identification of on- and off- centre brisk transient $(\mathrm{Y})$ cells in the cat retina. Proc R Soc Lond B Biol Sci 212:139-153.

Peichl L, Wassle H (1983) The structural correlate of the receptive field centre of alpha ganglion cells in the cat retina. J Physiol (Lond) 341:309-324.

Roberts ES, Huitron-Resendiz S, Taffe MA, Marcondes MC, Flynn CT, Lanigan CM, Hammond JA, Head SR, Henriksen SJ, Fox HS (2006) Host response and dysfunction in the CNS during chronic simian immunodeficiency virus infection. J Neurosci 26:4577-4585.

Rodieck RW, Watanabe M (1993) Survey of the morphology of macaque retinal ganglion cells that project to the pretectum, superior colliculus, and parvicellular laminae of the lateral geniculate nucleus. J Comp Neurol 338:289-303.

Sakai HM, Naka K, Korenberg MJ (1988) White-noise analysis in visual neuroscience. Vis Neurosci 1:287-296.

Shapley RM, Victor JD (1979) Nonlinear spatial summation and the contrast gain control of cat retinal ganglion cells. J Physiol (Lond) 290:141-161.

Shlens J, Field GD, Gauthier JL, Grivich MI, Petrusca D, Sher A, Litke AM, Chichilnisky EJ (2006) The structure of multi-neuron firing patterns in primate retina. J Neurosci 26:8254-8266.

Stafford DK, Dacey DM (1997) Physiology of the Al amacrine: a spiking, axon-bearing interneuron of the macaque monkey retina. Vis Neurosci 14:507-522.

Victor JD, Shapley RM (1979) The nonlinear pathway of Y ganglion cells in the cat retina. J Gen Physiol 74:671-689.

Volgyi B, Xin D, Amarillo Y, Bloomfield SA (2001) Morphology and physiology of the polyaxonal amacrine cells in the rabbit retina. J Comp Neurol 440:109-125.

Wassle H, Illing RB (1980) The retinal projection to the superior colliculus in the cat: a quantitative study with HRP. J Comp Neurol 190:333-356.

Wassle H, Peichl L, Boycott BB (1981) Morphology and topography of onand off-alpha cells in the cat retina. Proc R Soc Lond B Biol Sci 212:157-175.

White AJ, Sun H, Swanson WH, Lee BB (2002) An examination of physiological mechanisms underlying the frequency-doubling illusion. Invest Ophthalmol Vis Sci 43:3590-3599.

Yamada ES, Bordt AS, Marshak DW (2005) Wide-field ganglion cells in macaque retinas. Vis Neurosci 22:383-393. 